\title{
Lung Segmentation in Digital Radiographs
}

\author{
Ewa Pietka
}

\begin{abstract}
Computer-assisted interpretation of computer radiography $(C R)$ chest images including lung nodules detection, quantitative texture analysis, etc requires a lung delineation algorithm that restricts the area to be analyzed. This report presents a new lung-segmentation technique. It is performed in three phases. First, a histogram analysis finds a threshold value that eliminates the densest anatomic regions. Then, a gradient analysis separates the lungs from parts of thorax attached to the lungs that have not been removed in the previous phase. A smoothing routine yields the final image. By imposing a testing condition that results from the histogram analysis, underexposed images are not being considered. If being segmented, they exhibit a significant lung penetration. The test increases the accuracy of the procedure and makes it safer for an unsupervised application. The segmentation procedure has been implemented together with preprocessing functions in our clinical picture archiving and communication system.
\end{abstract}

Copyright 11994 by W.B. Saunders Company

KEY WORDS: computer radiography, chest radiographs, lung segmentation.

$\mathbf{P}$ ICTURE ARCHIVING and communication systems (PACS) used in clinics give the opportunity to develop but at the same time require more application software to be performed automatically. The PACS module handles images retrieved from different modalities including computer radiography (CR), computed tomography, magnetic resonance, ultrasound, and positron emission tomography, and yet, conventional radiography is the most common tool used in diagnostic radiology because of its simple implementation, use of inexpensive technology, and low $x$-ray exposure. Most of the $\mathrm{CR}$ radiographs are chest images. Many imageprocessing algorithms, including automated detection of lung nodules, ${ }^{1-4}$ quantitative texture analysis and characterization of interstitial disease, ${ }^{5-7}$ edge enhancement, ${ }^{8}$ and delineation of ribs $^{9,10}$ have already been developed. In all these applications, the information outside the lung region is irrelevant. Thus, a lung segmentation becomes the first image-processing procedure that has to be performed (automatically or manually) before any of the above mentioned techniques are applied.

Different computerized approaches to the segmentation problem have been reported over the years. There are methods considered as standards in the field of edge detection. Linear filtering followed by nonlinear decision-making processes seems to be the most frequently used. ${ }^{11}$ Beginning with Roberts or Sobel operators, ${ }^{12}$ radiologists have tried to solve the edge detection problem by applying a Gaussian function $^{13}$ or the Laplacian of Gaussian transformation. ${ }^{14}$ Petron and Kittler ${ }^{15}$ have derived filters for ramp edges of various slopes. Beside the lung contour, these methods also detect edges of all anatomic regions including ribs, arteries, blood vessels, and tubes (if any). This makes the lung-edge detection very difficult. Another global approach ${ }^{16}$ applies pattern recognition techniques in which each pixel is assigned to one of several anatomical regions (lungs, heart, subdiaphragm, arm, head, and background). The classification is based on three groups of features including gray-level based measures, local difference measures in a predefined neighborhood, and local texture measures. These measures are subjected to a classifier. Each pixel is considered as a separate object. A lack of context prevents the correct classification rate from exceeding $70 \%$ to $80 \%$.

In nonstandard approaches, the thresholding and histogram analyses ${ }^{17-19}$ have been used to enhance certain areas rather than to delineate anatomic regions. Another approach has been based on a set of predefined rules ${ }^{20}$ or anatomic constraint points ${ }^{21}$ to obtain each lung boundary. These rules rely on empirically determined assumptions about the relative size or area, accuracy of locating anatomic constraint points, etc. This causes a moderate success of these algorithms. Furthermore, thresholding techniques applied to an underexposed image either penetrate the lungs or retain parts of other anatomic regions yielding no reasonable results in any of these cases.

From the University Hospital of Geneva, Medical Imaging Unit, Geneva, Switzerland.

Address reprint requests to Ewa Pietka, PhD, University Hospital of Geneva, Medical Imaging Unit, 24, Rue Micheli-duCrest, CH-1211 Geneva, Switzerland.

Copyright 1994 by W.B. Saunders Company 0897-1889/94/0702-0011\$3.00/0 
In this study, a new lung delineation algorithm is presented. The analysis is based on a structural technique that uses both histogramthresholding analysis and gradient approach. No initial assumptions are made. First, a histogram is subjected to a test that detects underexposed images. Then, the first phase of the image analysis removes the densest parts, usually mediastinum, subdiaphragm, and parts of thorax (outside the lungs). It results in obtaining parts of lung edges. The already located edges are extended by using the gradient analysis over a limited image region. Finally, the smoothing procedure removes the noisy objects and detects the mediastinum edges.

\section{PARTIAL EDGE DETECTION}

The goal of this phase is to remove the densest regions and find separation areas between lungs and their background (ie, arms, head, and regions of thorax outside lungs). It can be noticed that, because of the thickness of the thorax and the location of the rib cage, very dense regions (apart from mediastinum and subdiaphragm) appear close to the edges of the lower parts of lungs. These regions can be eliminated by thresholding the image that yields parts of the lungs contour.

The threshold value is determined on the basis of a histogram analysis of a region called later a histogram region of interest (HROI). Its two edges (the top and the right edge) are located by profiles, extracted by the orientation procedure. ${ }^{22}$ They are the top of the shoulders and the location of the spine. The vertical profile that marks the spine is located in the densest areas of the mediastinum. In order not to consider the densest anatomic regions in the histogram analysis, this profile is shifted to the left to be scanned over the lungs (not the mediastinum). The lower edge is determined by scanning profiles over the entire image from the bottom of the image up toward the lung, and calculating their average densities. The largest decrease of the average value indicates the bottom of lungs and marks the bottom of the HROI. The left image edge serves also as a HROI edge.

These lines define the HROI (Fig 1A). It includes lungs, parts of thorax outside the lungs, and the shoulders. A histogram (Fig 1B) calcu-
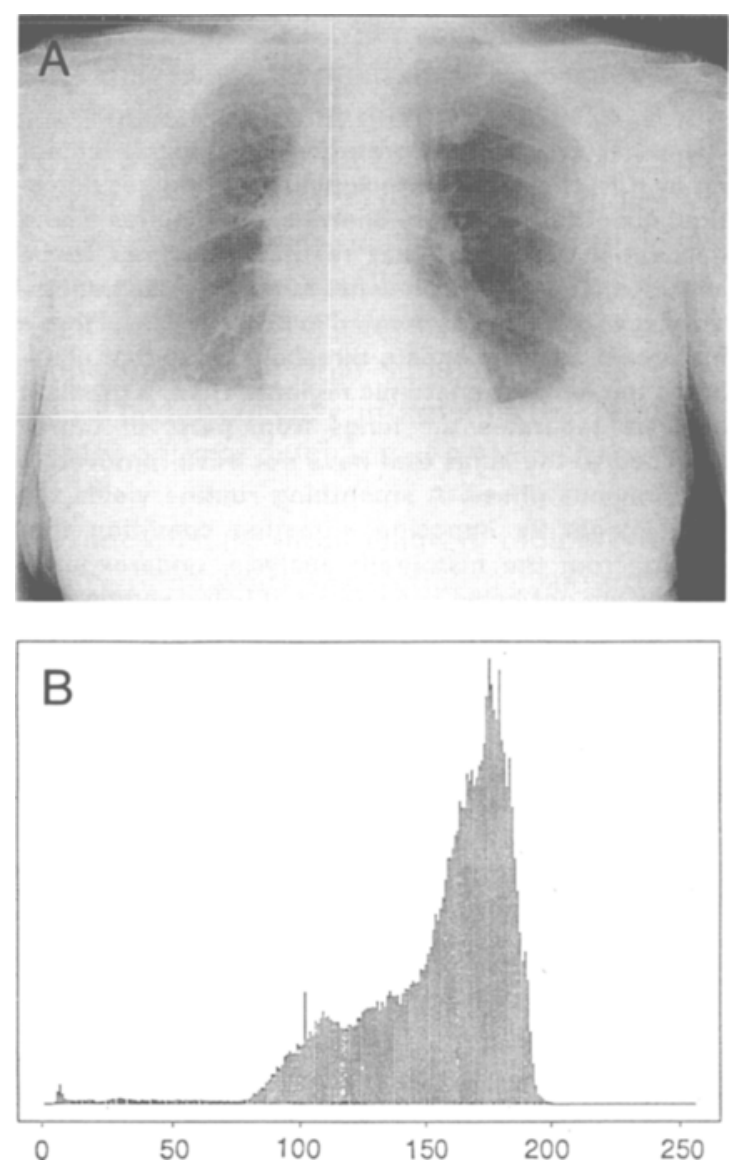

Fig 1. (A) Region of a radiograph selected for histogram analysis with (B) the corresponding histogram.

lated within this region is then searched for two waves. The lower-value wave (between 80 and 120 in Fig 1B) reflects the lung region, whereas the higher-value wave (between 150 and 190) reflects the densest parts of the HROI. In a regular, adult chest radiograph, the amplitude of the higher-value wave is $2 \pm 0.3$ times larger than the lower-value wave amplitude. In underexposed, contrast-lacking images, the ratio of both amplitudes drops to 1.2. This has been observed in some pediatric radiographs. A thresholding technique applied to these images penetrates the lungs. To successfully threshold the image, the ratio of these two maxima can not be smaller than 1.5 . If this condition is met, the histogram is searched for the breakpoint (the second derivative equal to 0 ) on the increasing slope of the higher-value wave. It serves as a threshold value.

In this application, the thresholding proce- 

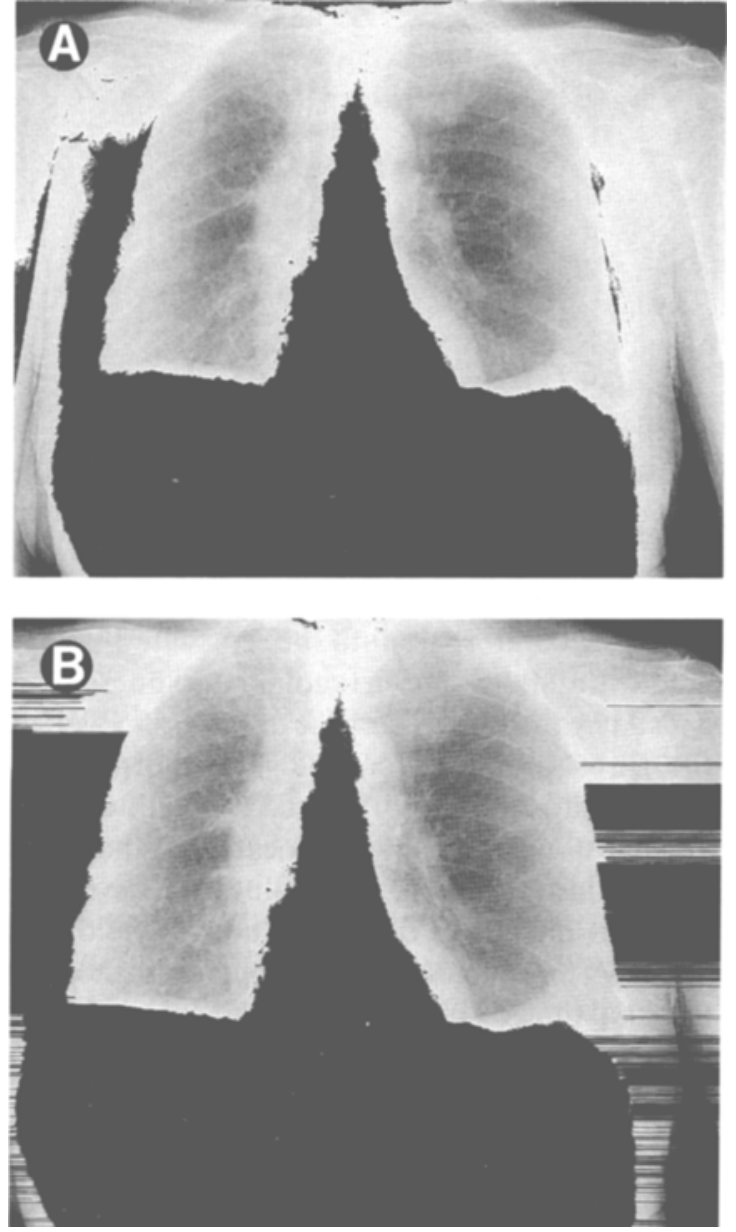

Fig 2. Lung image after (A) applying the thresholding procedure and $(B)$ the profile analysis.

dure (performed on the entire image) turns to zero all pixels above the threshold value, whereas it does not change the others. The densest regions including the mediastinum, subdiaphragm, parts of the arms, and thorax outside the lungs are eliminated (Fig 2A). However, it does not offer a complete removal of the entire tissue outside the lungs. Although a decrease of the threshold value reduces the unwanted areas, it also penetrates the lungs. Thus, this procedure yields a gray-scale image that includes the lungs and parts of their background.

The already removed parts of the thorax that are attached to the lung edges are located by scanning profiles from the bottom of the HROI (Fig 1) toward its top. In the left-lung analysis, they are scanned from the left edge of the image to the spine, whereas, in the right-lung analysis, they are scanned from the right edge of the image to the spine. Each profile is being analyzed in a context of its neighbor profiles. Let us start with a left-lung edge. In this case, only the region to the left of the mediastinum is examined. Each profile is searched for the longest zero-value plateau. The location of the end of this plateau (defined as its distance from the left edge of the image) is plotted versus the distance from the bottom of the HROI considered as a fiducial level (Fig 3). After a median filtering smoothes the function, it is searched for a sequence of pixels that reflects the left-lung edge (region between about 200 and 570 in Fig 3 ). In this analysis, the first derivative is used. Because this sequence is mapped versus the location within a defined region, it locates unambiguously the lung edge.

A similar analysis is performed to the right of the mediastinum. In this case, the distance function is calculated with respect to the right edge of the image. After parts of the edges have been located, all pixels outside these edges are turned to zero (Fig 2B).

\section{GRADIENT ANALYSIS}

Because parts of the lung edges are already located, the following step is performed on regions above and below the detected edges. These parts are subjected to a gradient analysis. Because of the texture of the lungs and the existence of ribs, blood vessels, and sometimes tubes, the kernel has to be chosen very carefully. If it is too small, the anatomic structure within the lungs adds unwanted details that make the

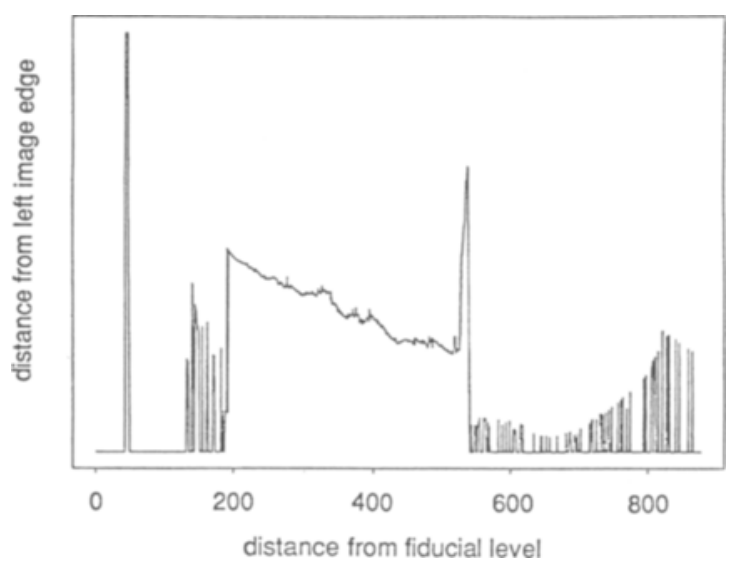

Fig 3. Function derived from the profile analysis. 
analysis more difficult. Besides, the line marking the lung edges is too thin and comparable with edges of other anatomic regions. On the other hand, a kernel that is too large increases the run time. Experimentally, the Sobel gradient operator has been chosen with a $7 \times 7$ kernel. The processed image is shown in Fig 4.

Further analysis starts from the top of the already detected edge. The following (located above) profiles of the gradient image are searched for the maximum. The assumption of the parabolic shape of the edges restricts the area to be analyzed. Again, the search for the lung edges is continued in both directions in the left and right lung region. After the edges are located, all pixels outside the edges are turned to 0 (Fig 5).

\section{SMOOTHING PROCESS}

Two different lung edges need to be smoothed: the external edge and the mediastinum edge. The external edge may include some spikes caused by the thresholding procedure. Because of the median filtering applied to the profile analysis function, they have not influenced the following steps. However, to obtain a smooth lung edge, they have to be corrected. Therefore, after calculating the function for the entire lung edge, the first derivative is searched for impulses that reflect edge points that need to be corrected. Then, a cubic spline interpolation procedure is applied to replace the spikes.

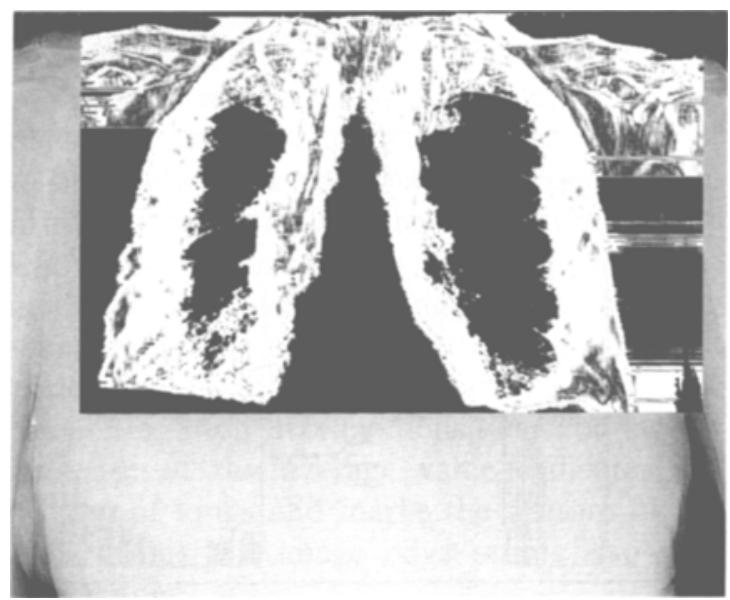

Fig 4. Chest radiograph processed with a $7 \times 7$ Sobel gradient operator.

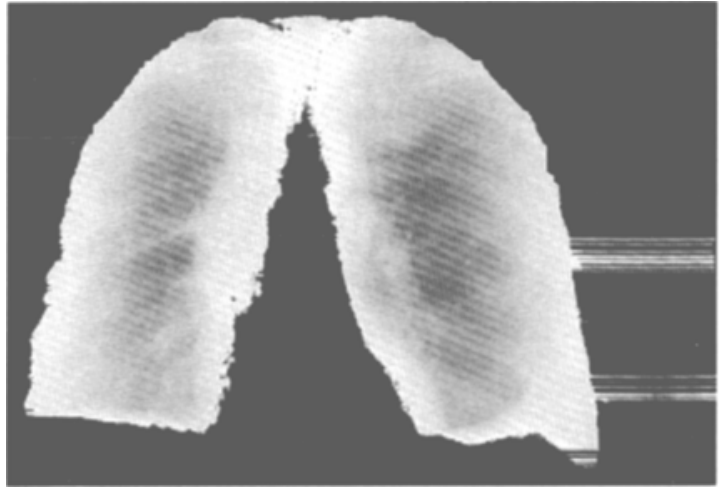

Fig 5. Lung segmentation after the gradient analysis has been applied.

The second region to be corrected is the mediastinum. Some irregular darker regions caused by the intervertebrae are not removed by the thresholding procedure. The morphologic erosion procedure, performed to finally smooth all edges, reduces the spots located on the top of mediastinum. Again, a profile analysis of the gradient image (as described above) yields the edges. A morphologic dilation operator smoothes the final image (Fig 6).

\section{RESULTS AND DISCUSSION}

Lung segmentation is a necessary step before any further image processing containing lung nodule detection or quantitative texture analysis can be applied without a manual selection of the region of interest. The analysis is performed in three steps. First, the densest regions of the image are removed. This yields parts of the lung

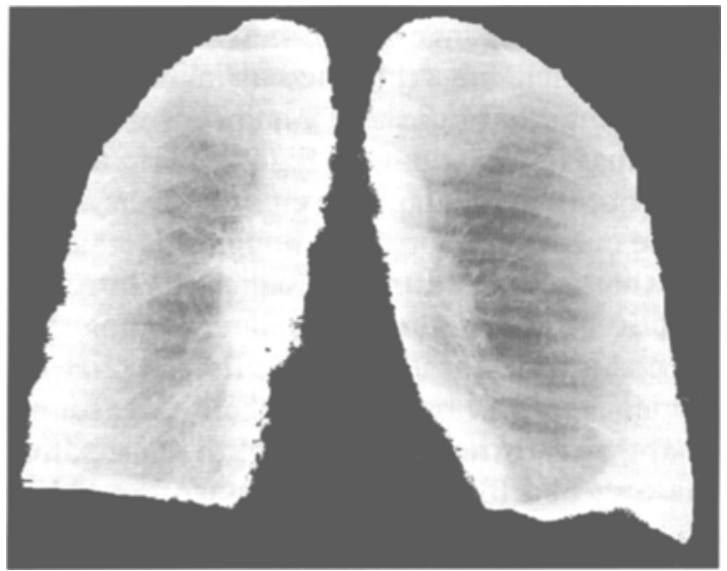

Fig 6. Final lung segmentation. 
edges. Then, the gradient analysis permits the edges to be extrapolated. Finally, a smoothing procedure eliminates noisy spikes attached to the external lung edges and spots from the mediastinum.

The algorithm has been subjected to a preliminary number of 40 clinical pediatric and adult CR chest radiographs. Because of further applications (mentioned above), the algorithm delineates lung regions correctly if no penetrations of lung are noticed and all anatomic regions (arms, head, abdomen) are removed. A correct delineation has been obtained in 35 images $(87.5 \%)$. The condition, imposed before thresholding the image to restrict the application of this algorithm has detected as underexposed 3 pediatric radiographs $(7.5 \%)$. All these radiographs lack contrast between the lungs and other anatomic regions, and the segmentation leads to a significant lung penetration. This test increases the accuracy of the analysis and makes the procedure safer for an unsupervised application. In the remaining 2 images (5\%), penetration of depth of less than $1 \mathrm{~cm}$ has been noticed along the ribs. The penetration area is restricted by the width of ribs.
This algorithm is being implemented together with preprocessing functions ${ }^{23}$ in our clinical PACS. Before being archived, the CR images are converted to a standard file format called Papyrus. $^{24}$ It is a unique file structure that handles information related to the patient, study, and images. It has been developed on the basis of the American College of Radiology/ National Electrical Manufacturers Association specification for image communication. The standardization also includes the image-orientation correction. Because the cassette can be placed in various orientation to accommodate the examination conditions, it becomes a major problem. A survey has shown ${ }^{22}$ that about $40 \%$ of images are not performed with the conventional image orientation. Therefore, it is necessary to develop an algorithm that identifies the current image orientation and, if it differs from the standard position viewed by radiologist, the image is rotated. ${ }^{22}$ Then, the lung-segmentation procedure is performed on a workstation equipped with two 2,048- $\times$ 2,560-pixel MegaScan monitors. It is followed by more advanced image-processing procedures including nodule enhancement and texture analysis.

\section{REFERENCES}

1. Lampeter W, Wandtke JC: Computerized searched of chest radiographs for nodules. Invest Radiol 21:384-390, 1986

2. Chiles C, Sherrier RH: Analysis if the solitary pulmonary nodule by means of digital techniques. J Thorac Imaging 5:55-60, 1990

3. Giger ML, Doi K, MacMahon $H$ : Image feature analysis and computer-aided diagnosis in digital radiography. Automated detection of nodules in peripheral lung fields. Med Phys 15:158-166, 1988

4. Giger ML, Ahn N, Doi K, MacMahon H: Computerized detection of pulmonary nodules in digital chest images: Use of morphological filters in reducing false-positive detections. Med Phys 17:861-865, 1990

5. Desaga JF, Dengler J, Wolf $\mathrm{T}$, et al: Filmdigitalisierung und Texturanalyse fur die digitale Klassifizierung von Lungenfleckschatten. Röntgen-Bl. 41:147-151, 1988

6. Katsuragawa $\mathrm{S}$, Doi $\mathrm{K}$, Nakamori $\mathrm{N}$, et al: Image feature analysis and computer-aided diagnosis in digital radiography: Effect of digital parameters on the accuracy of computerized analysis of interstitial disease in digital chest radiographs. Med Phys 17:72-78, 1990

7. Katsuragawa $S$, Doi $K$, Nakamori $N$, et al: Image feature analysis and computer-aided diagnosis in digital radiography: Detection and characterization of interstitial lung disease in digital chest radiographs. Med Phys 15:311319,1988
8. Daponte JS, Fox MD: Enhancement of chest radiographs with gradient operators. IEEE Trans Med Imaging 7:109-117, 1988

9. Sanada S, Doi K, MacMahon H: Image feature analysis and computer-aided diagnosis in digital radiography: Automated delineation of posterior ribs in chest images. Med Phys 18:964-971, 1991

10. Wechsler H, Sklansky J: Finding the rib cage in chest radiographs. Pattern Recognition 9:21-30, 1977

11. Davis LS: A survey of edge detection techniques. Comput Graphics Image Process 4:248-270, 1975

12. Gonzalez RC, Wintz P: Digital Image Processing (ed 2). Reading, MA, Addison-Wesley, 1987

13. Canny J: A computational approach to edge detection. IEEE Trans Pattern Anal Mach Intell 8:679-714, 1986

14. Sotak GE, Boyer KL: The Laplacian-of-Gaussian kernel: A formal analysis and design procedure for fast, accurate convolution and full-frame output. Comput Vision Graphics Image Processing 48:147-189, 1989

15. Petron M, Kittler J: Optimal edge detectors for ramp edges. IEEE Trans Pattern Anal Mach Intell 13:483-491, 1991

16. McNitt-Gray ME, Sayre JW, Huang HK: Pattern classification approach to chest radiographs. Radiology 185:301, 1992

17. Sherrier RH, Johnson GA: Regionally adaptive histo- 
gram equalization of the chest. IEEE Trans Med Imaging 6:1-7, 1987

18. Sezan MI, Tekalp AM, Schaetzing R: Automatic anatomically selective image enhancement in digital chest radiography. IEEE Trans Med Imaging MI 8:154-162, 1989

19. McNitt-Gray MF, Taira RK, Eldredge SE, Razavi M: Brightness and contrast adjustments for different tissue densities for digital chest radiographs. Proc SPIE 1445:468478,1991

20. Cheng D, Goldberg M: An algorithm for segmenting chest radiographs. Proc SPIE 1001:261-268, 1988
21. Turner AF, Kruger RP, Thompson WB: Automated computer screening of chest radiographs for pneumoconiosis. Invest Radiol 11:258-266, 1976

22. Pietka E, Huang HK: Orientation correction of chest images. J Digit Imaging 5:183-189, 1992

23. McNitt-Gray M, Pietka E, Huang HK: Image preprocessing for picture archiving and communication system. Invest Radiology 27:529-535, 1992

24. Ratib O, Appel R, Scherrer JR: Papyrus: A multimodality image file format for PACS and teleradiology. Radiology $177: 320,1990$ 